\title{
DENUMERABILITY CONDITIONS IN LOCALLY CONVEX VECTOR SPACES
}

\author{
JEAN DIEUDONNE
}

1. Let $E$ be a locally convex Hausdorff vector space, ${ }^{1} \mathrm{~J}$ its topology, $\mathfrak{B}$ a set of bounded subsets of $E$; we say that a subset $\mathfrak{F} \subset \mathfrak{B}$ is a fundamental system of sets of $\mathfrak{B}$ if any set of $\mathfrak{B}$ is contained in a set of $\mathfrak{F}$. When $\mathfrak{B}$ is the set of all bounded subsets of $E$, the condition that there exist a denumerable fundamental system of bounded sets has been extensively studied $[1 ; 3 ; 4]$ : coupled with mild restrictions on the topology $\mathrm{J}$, it narrows considerably the possibilities for $E$; as an example, let us only recall that if $J$ is metrizable, then the existence of a denumerable fundamental system of bounded sets implies that in fact $E$ is normable $[1$, p. 15 , exercise 20]. I propose to show in this note that even more stringent restrictions are imposed by the existence of a denumerable system $\mathfrak{F}$ when $\mathfrak{B}$ is either the set of all compact, or of all convex compact subsets of $E$.

2. We suppose first that there is a denumerable fundamental system of convex compact subsets in $E$. This implies that, on the dual $E^{\prime}$ of $E$, the topology $J_{c}^{\prime}$ of uniform convergence in the convex compact subsets of $E$ is metrizable, since a fundamental system of neighborhoods of 0 for $J_{c}^{\prime}$ consists of the polars of the convex compact sets of $E$. Moreover, as a convex compact subset of $E$ is also weakly compact, it follows from Mackey's first theorem [1, p. 68, Theorem 2] that when $E^{\prime}$ is given the topology $\Im_{c}^{\prime}$, its dual is $E$. However, the strong topology $\beta\left(E, E^{\prime}\right)$ on $E[1$, p. 93 , Exercise 7$]$ will in general be strictly finer than $J$, even if we suppose $J$ normable. An example of this is given by the space $E=R^{(N)}$ of sequences with only a finite number of nonzero terms, with the norm $\|x\|=\sup _{n}\left|x_{n}\right|$; it is known [1, p. 78, Exercise 3] that in that space every convex compact set is finite dimensional, hence there is a denumerable fundamental system of such sets. The dual $E^{\prime}$ is then the space $L^{1}(N)$ (space $l^{1}$ in Banach's terminology), but the topology $J_{c}^{\prime}$ on $E^{\prime}$ is the metrizable topology induced by the product topology on $R^{N}$; for that topology, $E^{\prime}$ is not complete, and in fact is dense in $R^{N}$; and it is easily seen that any bounded closed subset of $R^{N}$ is the closure of its intersection with $E^{\prime}$. Therefore the topology $\beta\left(E, E^{\prime}\right)$ is the finest locally convex topology on $E[1$, p. 94, Exercise 13].

However, we have the following

Received by the editors May 15, 1956.

1 We systematically use the results and terminology of [1] and [2]. 
Proposition 1. If $E$ is a $t$-space in which there is a denumerable system of convex compact subsets, then $E$ (with the topology $J$ ) is the strong dual of a space $F$ which is both a Fréchet and a Montel space.

Indeed, the bounded sets in $E^{\prime}$ for the topology $J_{c}^{\prime}$ are bounded for the coarser topology $\sigma\left(E^{\prime}, E\right)$, hence equicontinuous, since $E$ is a $t$-space; therefore the topology $\Im$ is the strong topology $\beta\left(E, E^{\prime}\right)$. But as $E^{\prime}$ (with $J_{c}^{\prime}$ ) is metrizable, hence a quasi-t-space, any bounded set in $E$ (for $J=\beta\left(E, E^{\prime}\right)$ ) is equicontinuous when $E$ is considered as the dual of $E^{\prime}[1$, p. 92, Exercise 6a], hence contained (by definition of $J_{c}^{\prime}$ ) in a convex compact subset of $E$. This shows that $E$ (with $J$ ) is a Montel space $[1$, p. 89 , Definition 5]; furthermore the topology $J_{c}^{\prime}$ is equal to the strong topology $\beta\left(E^{\prime}, E\right)$, hence $E^{\prime}$, with that topology, is also a Montel space [1, p. 90, Proposition 7]. But then a Cauchy sequence in $E^{\prime}$ (for $J_{c}^{\prime}$ ) is contained in a compact set, hence converges, which shows that $E^{\prime}$ is complete, in other words a Fréchet space, and this ends the proof.

We may observe that the converse of Proposition 1 is trivial, hence we have thus an intrinsic characterization of strong duals of FréchetMontel spaces.

3. Let us now suppose that there is a denumerable fundamental system $\left(A_{n}\right)$ of circled compact subsets in $E$ (when $E$ is complete or quasi-complete, this is equivalent to the previous assumption, but we do not at first assume any of these properties for $E$ ). On $E^{\prime}$, the topology $\Im_{k}^{\prime}$ of uniform convergence in the compact subsets of $E$ is then metrizable. Let $\hat{E}$ be the completion of $E, \widehat{J}$ the topology obtained from $\Im$ by completion; as $E^{\prime}$ can also be considered as the dual of $\hat{E}$, we can suppose $\hat{E}$ naturally imbedded in the algebraic dual $E^{\prime *}$ of $E^{\prime}$. Let $C_{n}$ be the closed circled convex hull of $A_{n}$ in $\hat{E}$; it is compact for $\hat{\mathrm{J}}$, hence also for the coarser topology $\sigma\left(\hat{E}, E^{\prime}\right)$. Therefore on $C_{n}$ the topologies induced by $\widehat{J}$ and $\sigma\left(\hat{E}, E^{\prime}\right)$ are the same; hence $C_{n}$ is the closed circled convex hull of $A_{n}$ in $E^{\prime *}$ for the topology $\sigma\left(E^{\prime *}, E^{\prime}\right)$. This proves $\left[1\right.$, p. 52, Proposition 3] that $C_{n}$ is the polar in $E^{\prime *}$ of the polar $A_{n}^{0}$ of $A_{n}$; as the $A_{n}^{0}$ form a fundamental system of neighborhoods of 0 in $E^{\prime}$ for $J_{k}^{\prime}$, we see that the dual of the metrizable space $E^{\prime}$ is the subspace $E_{1}$ of $\hat{E}$, union of the $C_{n}$. In general we will have $E_{1} \neq \hat{E}$, the strong topology $\beta\left(E_{1}, E^{\prime}\right)$ will be strictly finer than the topology $\Im_{1}$ induced by $\bar{\jmath}$, and the compact sets in $E_{1}$ for the topology $\Im_{1}$ will not be compact for $\beta\left(E_{1}, E^{\prime}\right)$.

Let $F=E^{\prime}$ with the topology $J_{k}^{\prime}$, and consider its completion $\hat{F}$, which is therefore a Fréchet space; $E_{1}$ is also the dual of $\hat{F}$, and the sets $C_{n}$ are still the polars of a fundamental system of neighborhoods 
of 0 in $\hat{F}$. Consider on $E_{1}$ the topology $\Im_{k}$ of uniform convergence in the compact subsets of $\hat{F}$; any compact set in $E_{1}$ for $J_{k}$ is also equicontinuous $\left[1\right.$, p. 65 , Theorem 1] hence contained in one of the $C_{n}$; moreover, on $C_{n}$ the topology induced by $J_{k}$ is the same as the topology induced by the weak topology $\sigma\left(E_{1}, \hat{F}\right)[1$, p. 23, Proposition 5], and therefore the $C_{n}$ form again a fundamental system of compact subsets for $J_{k}$. Moreover, $J_{k}$ is finer than the topology $J_{1}$; for if $U$ is a neighborhood of 0 in $E_{1}$ (or in $E$ ) for the latter topology, its polar $U^{0}$ in $\hat{F}$ is compact for the topology of uniform convergence in the $C_{n}$ $[1$, p. 23, Proposition 5].

Summing up, we can therefore describe the general situation as follows: take a Fréchet space $G$, and consider its dual $G^{\prime}=E_{1}$; the topology $J_{1}$ must be a topology coarser than the topology $J_{k}$ of uniform convergence in the compact sets of $G$, and finer than some topology $\sigma\left(E_{1}, F\right)$, where $F$ is a dense subspace of $G$; moreover $E$ must be a subspace of $E_{1}$, dense for $J_{1}$, and such that any compact set in $E$ should be equicontinuous (in $E_{1}$, considered as dual of $G$ ). Whether these conditions are sufficient, when $E \neq E_{1}$, to insure that there will be a denumerable system of compact subsets of $E$, I do not know; actually, I have no example in which $E \neq E_{1}$. If we restrict ourselves to the case $E=E_{1}$, the preceding conditions are sufficient, since the polar $C_{n}$ of a neighborhood of 0 in $G$ is compact for $\sigma\left(E_{1}, G\right)$, and by definition of $J_{1}$, the topology induced on $C_{n}$ by $J_{1}$ is identical to the one induced by $\sigma\left(E_{1}, G\right)[1$, p. 23, Proposition 5]. However, this cannot be considered as an explicit description of the admissible topologies $J_{1}$. All I can do in this direction is to give the rather obvious following sufficient condition, namely that $F$ be a $t$-space (which, as is well known, does not imply that $F=G$, nor even that $F$ be a Baire space). Indeed, if that condition is satisfied, a compact set in $E_{1}$, for the topology $J_{1}$, is bounded for that topology, hence also for the coarser topology $\sigma\left(E_{1}, F\right)$; but as $E_{1}$ is the dual of $F$ and $F$ is a $t$-space, any set bounded for $\sigma\left(E_{1}, F\right)$ is equicontinuous [1, p. 65, Theorem 1].

That it does not seem possible to relax very much the preceding condition on $F$ is seen by returning to the example in $\$ 2$, considered under our present point of view. We take for $G$ the Fréchet space $R^{N} ; E_{1}=G^{\prime}$ is therefore the space $R^{(N)}$, and here $J_{k}$ is the finest locally convex topology on that space. We next take $F=L^{1}(N)$ (with the topology induced by the product topology on $G$, that is the weak topology $\sigma\left(F, E_{1}\right)$, and not the norm topology). The norm topology on $E_{1}$ is exactly the Mackey topology $\left[1\right.$, p. 70] $\tau\left(E_{1}, F\right)$, since it is a metrizable topology and $F$ is the dual of $E_{1}$ for that topology [1, p. 71, Proposition 6]. However, equicontinuous sets in $E_{1}$ are finite dimen- 
sional, and there are nonfinite dimensional compact subsets for the norm topology (for instance, sequences converging to 0 ).

4. Let us now return to the problem as formulated at the beginning of $\S 3$, and see what happens when we impose additional requirements on the topology $\mathfrak{J}$ of $E$. Suppose first that $J$ is the Mackey topology $\tau\left(E, E^{\prime}\right)=\tau(E, F)$; then the topology $J_{1}$ on $E_{1}$ is also the Mackey topology $\tau\left(E_{1}, F\right)[1$, p. 79, Exercise $5 \mathrm{c}]$. The polars of the neighborhoods of 0 for $J_{1}$ are therefore the circled convex subsets of $F$ which are compact for the weak topology $\sigma\left(F, E_{1}\right)$; we have already seen that these sets are compact for the topology $\Im_{k}^{\prime}$. Conversely, if a subset $K$ of $F$ is convex and compact for $J_{k}^{\prime}$, it is also compact for the coarser topology $\sigma\left(F, E_{1}\right)$. It is easy to give examples of Fréchet spaces which are not Montel spaces, and in which weakly compact sets are also compact, for instance the space $L^{1}(N)$ (with its norm topology; cf. [1, p. 118, Exercise 4b); in that case $E_{1}=L^{\infty}(N)$ is the space of bounded sequences (space $m$ in Banach's terminology), and the balls of radius $n$ in $L^{\infty}(N)$ form a fundamental system of compact subsets for the topology $\tau\left(E_{1}, F\right)$.

We observe that in the preceding example, $E_{1}$ is not a quasi-t-space $[1$, p. 95, Exercise 20a]. All quasi-t-spaces known at present are either bornological or $t$-spaces; for the two latter categories, we have a much more precise result:

Proposition 2. If $E$ is a bornological space or a $t$-space in which there is a denumerable fundamental system of compact subsets, then $E$ is dense in the strong dual $E_{1}$ of a Fréchet-Montel space.

Suppose first that $E$ is bornological; then [1, p. 37, Exercise 18], $F$ is complete for $J_{k}^{\prime}$, hence a Fréchet space. Furthermore, as $J$ is the Mackey topology [1, p. 71, Proof of Proposition 6], $J_{1}$ is also the Mackey topology $\tau\left(E_{1}, F\right)$ as seen above, and as $F$ is complete, closed convex hulls of compact sets of $F$ are again compact; as conversely convex weakly compact sets of $F$ are compact for $J_{k}^{\prime}$, we see that $J_{1}$ is identical to the topology $J_{k}$. Therefore, as $F$ is bornological for $J_{k}^{\prime}, E_{1}$ is complete for $J_{1}[1$, p. 37, Exercise 18], hence a $t$-space for that topology, as the completion of a bornological space $[1$, p. 13 , Exercise 12c]. But then, bounded sets in $F$ (for $J_{k}^{\prime}$ ) are equicontinuous, hence weakly relatively compact, hence relatively compact for $J_{k}^{\prime}$, and $F$ is a Montel space; moreover the topology $J_{k}=J_{1}$ on $E_{1}$ is then the strong topology $\beta\left(E_{1}, F\right)$.

Suppose now that $E$ is a $t$-space; then $E_{1}$ (with $J_{1}$ ) is also a $t$-space, for if $T$ is any barrel in $E_{1}, T \cap E$ is a barrel in $E$, hence a neighborhood of 0 for $\mathfrak{J}$, and the closure of $T \cap E$ in $E_{1}$, which is contained in 
$T$, is a neighborhood of 0 for $\Im_{1}$. It follows as above that in $F$ the bounded sets for $\Im_{k}^{\prime}$ are weakly relatively compact, and therefore $F$ is semi-reflexive $[1$, p. 88 , Theorem 1$]$; but as $F$ is metrizable, it is then necessarily complete. The topology $J$ being again the Mackey topology [1, p. 70, Proposition 5], the argument is then concluded as above.

I do not know if the conclusion of Proposition 2 still holds if $E$ is only supposed to be a quasi- $t$-space; and, as said before, I have no example of the situation of Proposition 2 in which $E \neq E_{1}$. For $E=E_{1}$, the converse of Proposition 2 is of course true, since it is known that the strong dual of a Fréchet-Montel space is bornological, by a result of Grothendieck [1, p. 97, Exercise 22c].

\section{An interesting consequence of Proposition 2 is the following}

Proposition 3. If in a metrizable locally convex space $E$ there is a denumerable fundamental system of compact subsets, then $E$ is finite dimensional.

For $E$ is bornological, and therefore $E_{1}$ is a Montel space for $J_{1}$ by Proposition 2. On the other hand, as $E$ is metrizable, so is $E_{1}$; but the strong dual of a Fréchet space $F$ can only be metrizable if $F$ is a Banach space $\left[1\right.$, p. 15, Exercise 20], and then $E_{1}$ is also a Banach space. But a Banach space which is also a Montel space is finite dimensional.

There is perhaps some interest in the following more direct proof of Proposition 3. Suppose first that $E$ is complete, hence a Fréchet space: being by assumption the union of a denumerable sequence $\left(K_{n}\right)$ of compact sets, it follows from Baire's theorem that one of the $K_{n}$ must have an interior point, and the existence in $E$ of a relatively compact nonempty open set implies that $E$ is finite dimensional. If now $E$ is not complete, we consider its completion $\hat{E}$; the result will follow if we prove that in $\hat{E}$ there is a denumerable fundamental system of compact subsets. But this results from the fact that every compact set in $\hat{E}$ is contained in the closed convex hull of a sequence of points of $E$ converging to 0 , a property which is itself an immediate consequence of $[1$, p. 73 , Proposition 7] and of the fact that in the dual $E^{\prime}$ of $E$, equicontinuous subsets are the same whether one considers $E^{\prime}$ to be the dual of $E$ or of $\hat{E}$, and on these sets the topologies induced by $\sigma\left(E^{\prime}, E\right)$ and $\sigma\left(E^{\prime}, \hat{E}\right)$ are the same.

\section{BIBLIOGRAPHY}

1. N. Bourbaki, Eléments de mathématique, Livre V: Espaces vectoriels topologiques, Chaps. III-V, Actualités Scientifiques et Industrielles, no. 1229, Paris, Hermann, 1955. 
2. J. Dieudonne, Recent developments in the theory of locally convex vector spaces, Bull. Amer. Math. Soc. vol. 59 (1953) pp. 495-512.

3. G. W. Mackey, On infinite-dimensional linear spaces, Trans. Amer Math Soc. vol. 57 (1945) pp. 155-207.

4. - On convex topological linear spaces, Trans. Amer. Math. Soc. vol. 60 (1946) pp. 519-537.

NORTHWESTERN UNIVERSITY

\section{A NOTE ON THE CONTINUITY OF THE INVERSE}

\section{ROBERT ELLIS}

In his article [2] Wallace mentions the following problem: let $X$ be an algebraic group with a locally compact Hausdorff topology such that the map of $X \times X$ into $X$ which takes $(x, y)$ into $x y$ for all $x, y \in X$ is continuous. Then is $X$ a topological group? The purpose of this note is to answer this question in the affirmative.

Lemma 1 is an immediate consequence of the continuity of multiplication, and the proof of Lemma 2 appears in [1]. The proofs of these lemmas will therefore be omitted.

Lemma 1. Let $F$ be a filter on $X$ such that $F \rightarrow x$ and $F^{-1} \rightarrow y$. Then $y \equiv x^{-1}$.

Lemma 2. Let $A$ be a compact subset of $X$. Then $A^{-1}$ is closed.

Lemma 3. Let $E$ be a countable subset of $X$, and let $x$ be a limit point of $E$. Then $x^{-1}$ is a limit point of $E^{-1}$.

Proof. There is an ultra filter base $\mathcal{U}$ on $E$ such that $\mathfrak{u} \rightarrow x$. By Lemma 1 it is sufficient to show that there is $y \in X$ such that $\mathfrak{u}^{-1} \rightarrow y$. To this end it will be shown that there is a compact set $C$ and a set $U \in \mathcal{U}$ such that $U^{-1} \subset C$.

Let $B=E \cup\{x\}$ and $D=\cup_{n=-\infty}^{\infty} B^{n}$. Then $D$ is a countable subgroup of $X$. Furthermore, if $A=\bar{D}$, then the continuity of multiplication implies that $A^{2} \subset A$.

Now let $V$ be a compact neighborhood of the identity. Then $\bar{D}=A$ implies that $A \subset D V^{-1}$. Thus $A=\mathrm{U}\left[d V^{-1} \cap A \mid d \in D\right]$ $=U\left[d\left(V^{-1} \cap A\right) \mid d \in D\right]$ since $D$ is a group and $A^{2} \subset A$. But $d\left(V^{-1} \cap A\right)$

Received by the editors April 14, 1956. 